\title{
Indoor and Outdoor Air Quality and Its Relation to Allergic Diseases among Children: A Case Study at a Primary School in Korea
}

\author{
Ho-Hyun Kim, Chang-Soo Kim ${ }^{1)}$, Young-Wook Lim, Min-A Suh ${ }^{1)}$ and Dong-Chun Shin ${ }^{1) * *}$ \\ The Institute for Environmental Research, Yonsei University College of Medicine, Seoul, Korea \\ ${ }^{1)}$ Department of Preventive medicine, Yonsei University College of medicine, Seoul, Korea
}

*Corresponding author. Tel: +82-2-2228-1869, E-mail: dshin5@yuhs.ac

\begin{abstract}
The purpose of this study is to investigate allergic diseases related to allergy caused by the exposure to indoor and outdoor sources of air pollution in primary schools. The symptoms questionnaire of allergic diseases based on the International Study of Asthma and Allergies in Childhood (ISAAC) was completed by the participants. The past and present status of asthma, allergic rhinitis, eczema, and allergic conjunctivitis were investigated by providing a questionnaire to all the participating children. Questionnaires were sent to a total of 61,350 children from 438 primary schools. A total of 40,522 children responded to the questionnaire, which represents a $66.1 \%$ return rate. Volatile Organic Compounds (VOCs), Aldehydes, and Particulate Matter $\left(\mathrm{PM}_{10}\right)$ were measured and analyzed from October to December of 2006, in 82 primary schools. The final study population comprised 35,168 children with complete data which excluded incomplete questionnaire responded by 5,354 children. Based on the survey, the level of indoor air contamination did not appear to be high, but $27.2 \%$ of the schools evaluated had exceeded the $\mathrm{PM}_{10}$ level specified by the school health guidelines $\left(100 \mu \mathrm{g} / \mathrm{m}^{3}\right)$. The overall mean concentration of formaldehyde was $22.07 \mu \mathrm{g} / \mathrm{m}^{3}$ and $1.0 \%$ of schools ( $1 \mathrm{school}$ ) exceeded the $100 \mu \mathrm{g} / \mathrm{m}^{3}$. Statistically significant relationships have been observed between indoor air quality and prevalence rate of allergic rhinitis and conjunctivitis of primary schools in Korea.
\end{abstract}

Key words: Allergic diseases, ISAAC, Primary school children, VOCs, Aldehydes, $\mathrm{PM}_{10}$

\section{INTRODUCTION}

In the International Study of Asthma and Allergies in Childhood (ISAAC) research report, the highest asthma prevalence occurred in industrialized and western- ized countries (Ho et al., 2007). For young individuals, schools represent the environment where they pass a substantial portion of the day (Silvers et al., 1994). Other studies confirm that indoor air quality in schools is far from what may be characterized as a "healthy microenvironment" (Siskos et al., 2001; Hirsch et al., 1999; Knox et al., 1997). A number of studies have revealed that school air may be a source of a wide spectrum of air pollutants, such as VOCs, etc (Braniš et al., 2005).

Asthma is a chronic inflammatory disorder of the airways characterized by episodes of recurrent wheezing, shortness of breath, chest tightness, and cough (Downs et al., 2001). Atopic dermatitis (AD), a common chronic inflammatory skin disease, is one of the most common disorders in children (Lee et al., 2001). Currently, allergic diseases are considered to be related to environmental problems. These are associated with "problem buildings" caused by moving into new buildings, and "Sick School Syndrome" caused by contact with chemical substances in newly built school. These syndromes develop primary school students who spend most of the day in primary school area were easy to develop a symptoms so-called "sensitive group". Recurrent sneezing due to viral infection has potential to cause sickness in many preschool children, but may not necessarily represent a respiratory symptom. Especially when there is no medical or family history of asthma or eczema, these situations are known as 'viral-induced' sneezing episode. These symptoms are different from those in children with asthma since acute symptoms occurring by certain interval may be related to exercise, environmental stimuli (such as pollen, smoke, and air pollution) and contact with animals (Strachen and Gerritsen, 1996).

Increased exposure to air polluting substances arising from both indoors and outdoors, such as particulate matter $\left(\mathrm{PM}_{10}\right)$, Volatile Organic Compounds (VOCs), Formaldehydes (HCHO), Ozone $\left(\mathrm{O}_{3}\right)$, Nitrogen dioxides $\left(\mathrm{NO}_{2}\right)$ and the emission gases of diesel vehicles along with increased time spent indoors have increased 
the risk of exposure to various antigens (Ho et al., 2007). This is also thought to be a major reason for the increasing incidence of asthma, pulmonary and allergic diseases (Breysse et al., 2005; Nielsen et al., 2005; Delfino, 2002; Nielsen et al., 2002; Pandya et al., 2002). An increased prevalence of allergic diseases has been found in urban areas of industrialized countries that experience heavy traffic (Brunekreef et al., 1997; Weiland et al., 1994). Moreover, increasing allergic sensitization is being detected in individuals living in heavily polluted areas (Nicolai et al., 2003; Wyler et al., 2000; Popp et al., 1989). According to a 5-year investigation of allergic diseases conducted by the Korean Academy of Pediatric Allergy and Respiratory Diseases since 1995, the asthma prevalence rate in primary school students has increased from $7.7 \%(25,361$ tested) in 1995 to $9.1 \%$ (28,050 tested) in 2000 (MOHW, 2005).

The aim of this study is to determine the effect of school indoor air quality to the allergic diseases of primary school children through the analyses of various chemical substances.

\section{METHODS}

\section{1 Study Subjects}

Data on geographic area and number of students of all primary schools $(n=6,279)$ in 2006 were acquired from ministry of education, science and technology (MEST) of the Republic of Korea.

We exclude [Closed school] or [Temporarily closed school] in 2006 and remote island such as Jeju. Remaining schools were separated based on the ratio of [Metropolitan city]-[Mid to small city]-[Other Gun, Myun, and Eup]. Randomized sample selection criteria were described as follows: 1) school with more than 100 students (i.e. more than three classrooms), 2) private schools included unconditionally since most Korean schools are national or public schools; and 3) determine the ratio of [Metropolitan city]-[Middle sized city]-[Other Gun, Myun, and Eup]. After the initial round of selection, the following two conditions were evaluated: Inclusion of more than 70 primary schools near major industrial complexes around the nation, and the time of school was built ([2-5 years], [6-10 years], [11-20 years], [More than 21 years]). Among selected primary schools, 3rd, 4th, and 5th grade students mainly participated to the survey.

Letters of invitation, informed consent, and ISAAC questionnaires were mailed to 61,350 children from October through November 2006. All mailing contents were administered to the parents of the children. Among 61,350 subjects of 443 primary schools, a total of
$40,522(66.1 \%)$ of 438 primary schools responded the mailing survey.

\section{2 Sampling and Analysis}

Among the schools that participated in the first survey, 82 primary schools were selected according to the school selection criteria. The detailed sample selection criteria for the investigation of indoor environmental status were 1) school size with more than 100 students (Namely, more than 3 classrooms), 2) private schools are included unconditionally, 3) considering the ratio of [Metropolitan city]-[Middle sized city][Other Gun, Myun, and Eup], 4) evaluating the adequacy of following two conditions after sampling in case of unsatisfaction, 5) Inclusion of more than 10 primary schools near major industrial complexes around the nation, 6) Considering the establishment year of schools as the ratio of [School built year], [2-5 years], [6-10 years], [11-20 years], [More than 21 years].

The standardized as well as widely adopted International Study of Asthma and Allergies in Childhood (ISAAC) questionnaire was utilized to investigate the history and prevalence rate of allergic disease such as asthma, atopy dermatitis, allergic rhinitis and conjunctivitis (Lee et al., 2001; ISAAC Steering Committee, 1998; Asher et al., 1995). In this study, Korean version of ISAAC questionnaire for asthma following the guidelines suggested by ISAAC was used. Detailed characteristics of the Korean version of ISAAC questionnaire have been reported previously (Yeon et al., 2005; Oh et al., 2003).

In addition, questions on living environment such as monthly electricity bill (as a proxy indicator of socio-economic status), residence type, age of building, and history of moving to new house were added. The questionnaires were distributed to each school, and students were advised to fill out the questionnaire with the help of parents.

This study was approved by the Ethics Committee of Yonsei University, College of Medicine and informed consent was obtained from all the parents and principals of primary schools.

The analysis of indoor air was conducted between October and December of 2006 from the final group of selected primary schools. Measurements were taken in classrooms, hallways, and the outdoors. Aldehydes (1 hr) (Formaldehyde, Acetaldehyde), VOCs (1 hr) (Benzene, Toluene, Ethylbenzene, Xylene), and $\mathrm{PM}_{10}$ $(8 \mathrm{hr})$ were quantified from each locations. Sampling was performed during class hours, and the classrooms examined were notified not to allow ventilation on the day of sample collection. Five repeated recovery ratios of VOCs and aldehyde were averaged, which resulted 85 to $110 \%$ satisfactorily. 


\section{3 Statistical Analysis}

For the analyses, we excluded 5,354 children whose data was uncompleted. The final study population comprised 35,168 children with complete data. Data were analyzed by residential regions which were four regions (Rural/Industrial complex/Metropolitan/ Middle-sized cities). The difference of allergic disease by regional communities and multiple group comparison analysis was conducted using ANOVA. T-test was performed to compare the aldehydes, VOCs and $\mathrm{PM}_{10}$ level of two groups (case and control) and a p-value less than 0.05 was considered statistically significant. All statistical analyses were performed using the SAS 9.1 statistical package.

\section{RESULTS AND DISCUSSION}

\section{1 Prevalence of Allergic Diseases}

The survey was separated into four regions: rural, industrial complexes, metropolitan cities, and middlesized cities. The house types were most apartments (above 60\%) and there were single and multi-detached houses. Houses were for the most part constructed more than 5 years ago (above $70 \%$ ). Overall, $30 \%$ contained indoor-smoker. In addition, a higher proportion of households had ventilation (above 95\%). Average ventilation was 1-2 times per day. The ratio of girl (56\%) was slightly higher than boy (44\%).
The distribution of allergic diseases is provided in Table 1 . For asthma, $10.8 \%$ of students responded to have symptoms of wheezing; whereas only $9.3 \%$ students in rural regions reported wheezing symptoms since birth. The overall percentage of students diagnosed with asthma was $7.7 \%$. The children from rural regions demonstrated the lowest ratio for prevalence of diagnosed with asthma for lifetime (6.5\%) compared to industrial complexes (8.9\%), metropolitan cities $(8.0 \%)$, and middle-sized cities $(7.7 \%)$. The prevalence of asthma increased from $7.7 \%$ in 1995 to $9.1 \%$ in 2000 (Hong et al., 2004). It is, however, important to note that the variation may arise in the survey due to the use of different institutions compromising students with different age distribution at 1-6 grades.

The overall lifetime prevalence of allergic rhinitis was $39.5 \%$. Industrial complex regions had a higher 12-month prevalence rate of rhinitis (38.4\%) than other regions while the lowest number of students from rural regions $(27.1 \%)$. In rural regions, both the number of students diagnosed and showed symptoms of rhinitis in the past year was lower compared to other regions. Rhinitis symptoms which included sneezing, congestion and nose itching, appeared most frequently in March, April (spring), and September, October (fall), (data not shown). Congestion (23.6\%) was the most prevalent rhinitis symptom and $12.9 \%$ of the symptoms were found to be itching at the nose (data not shown).

Allergic conjunctivitis showed a similar pattern, as

Table 1. Prevalence of symptoms of allergic diseases.

(Unit: person $(\%)$ )

\begin{tabular}{|c|c|c|c|c|c|c|c|c|c|c|c|}
\hline \multirow{2}{*}{ Asthma } & \multicolumn{2}{|c|}{$\begin{array}{c}\text { Total } \\
(\mathrm{N}=35168)\end{array}$} & \multicolumn{2}{|c|}{$\begin{array}{c}\text { Rural } \\
(\mathrm{N}=6725)\end{array}$} & \multicolumn{2}{|c|}{$\begin{array}{l}\text { Industrial } \\
\text { complex } \\
(\mathrm{N}=3318)\end{array}$} & \multicolumn{2}{|c|}{$\begin{array}{c}\text { Metropolitan } \\
\text { cities } \\
(\mathrm{N}=13621)\end{array}$} & \multicolumn{2}{|c|}{$\begin{array}{c}\text { Middle } \\
\text { sized cities } \\
(\mathrm{N}=11504)\end{array}$} & \multirow[t]{2}{*}{ p-value ${ }^{a}$} \\
\hline & & & & & & & & & & & \\
\hline Symptom lifetime & 3640 & $(10.8)$ & 597 & $(9.3)$ & 382 & $(12.0)^{*}$ & 1508 & (11.4) & 1153 & $(10.5)$ & $<0.0001$ \\
\hline Symptom-last 12 months & 1669 & (4.9) & 296 & $(4.6)$ & 187 & $(5.9)^{*}$ & 660 & $(5.0)$ & 526 & $(4.8)$ & $<0.0001$ \\
\hline Diagnostic lifetime & 2709 & $(7.7)$ & 437 & $(6.5)$ & 294 & $(8.9)^{*}$ & 1093 & $(8.0)$ & 885 & (7.7) & $<0.0001$ \\
\hline Treatment, last 12 months & 891 & $(2.5)$ & 156 & $(2.3)$ & 102 & $(3.1)$ & 355 & $(2.6)$ & 278 & $(2.4)$ & 0.1068 \\
\hline \multicolumn{12}{|l|}{ Allergic rhinitis } \\
\hline Symptom lifetime & 13588 & $(39.5)$ & 2150 & $(32.8)$ & 1420 & $(43.8)^{*}$ & 55595 & $(41.8)$ & 4423 & $(39.3)$ & $<0.0001$ \\
\hline Symptom-last 12 months & 11703 & $(34.0)$ & 1774 & $(27.1)$ & 1246 & $(38.4)^{*}$ & 4866 & $(36.4)$ & 3817 & (33.9) & $<0.0001$ \\
\hline Diagnostic lifetime & 9926 & $(28.2)$ & 1326 & (19.7) & 1090 & $(32.9)^{*}$ & 4237 & (31.1) & 3273 & $(28.5)$ & $<0.0001$ \\
\hline Treatment, last 12 months & 7597 & (21.6) & 1029 & $(15.3)$ & 874 & $(26.3)^{*}$ & 3179 & $(23.3)$ & 2515 & (21.9) & $<0.0001$ \\
\hline \multicolumn{12}{|l|}{ Eczema } \\
\hline Symptom lifetime & 7608 & $(22.1)$ & 1170 & $(17.8)$ & 843 & $(26.0)^{*}$ & 3114 & $(23.3)$ & 2481 & $(22.0)$ & $<0.0001$ \\
\hline Symptom-last 12 months & 5637 & (16.4) & 855 & $(13.0)$ & 629 & $(19.4)^{*}$ & 2305 & $(17.2)$ & 1848 & (16.4) & $<0.0001$ \\
\hline Diagnostic lifetime & 10028 & $(28.5)$ & 1419 & $(21.1)$ & 1086 & $(32.7)^{*}$ & 4220 & $(31.0)$ & 3303 & $(28.7)$ & $<0.0001$ \\
\hline Treatment, last 12 months & 4893 & $(13.9)$ & 774 & $(11.5)$ & 545 & $(16.4)^{*}$ & 1940 & $(14.2)$ & 1634 & $(14.2)$ & $<0.0001$ \\
\hline \multicolumn{12}{|l|}{ Allergic conjunc-tivitis } \\
\hline Symptom lifetime & 6327 & $(18.4)$ & 979 & $(15.0)$ & 690 & $(21.3)^{*}$ & 2586 & $(19.4)$ & 2072 & $(18.4)$ & $<0.0001$ \\
\hline Symptom-last 12 months & 4816 & $(14.0)$ & 699 & $(10.7)$ & 549 & $(17.0)^{*}$ & 1999 & $(15.0)$ & 1569 & (13.9) & $<0.0001$ \\
\hline Diagnostic lifetime & 6778 & (19.3) & 901 & (13.4) & 825 & $(24.9)^{*}$ & 2813 & $(20.7)$ & 2239 & (19.5) & $<0.0001$ \\
\hline Treatment, last 12 months & 4076 & $(11.6)$ & 546 & $(8.1)$ & 505 & $(15.2)^{*}$ & 1673 & $(12.3)$ & 1352 & $(11.8)$ & $<0.0001$ \\
\hline
\end{tabular}

${ }^{\mathrm{a} A N O V A}$ test 
other diseases, having the lowest occurrence in students who experienced the conjunctivitis symptoms once during their lifetime or during the last one year in rural regions. Moreover, $19.3 \%$ of students were diagnosed with allergic conjunctivitis.

The overall lifetime prevalence of eczema symptom was $22.1 \%$, while the overall 12 -month prevalence of eczema was $16.4 \%$. Rural regions had a lower prevalence rate of diagnosed with eczema $(21.1 \%)$ than other regions.

\section{2 Indoor Air and Outdoor Quality of Primary Schools}

The indoor air results for the classrooms, hallways, and outdoor areas of primary schools around the country are summarized in Table 2.

The overall mean concentration of formaldehyde was $22.07 \mu \mathrm{g} / \mathrm{m}^{3}$ and $1.0 \%$ of schools ( 1 school) exceeded the $100 \mu \mathrm{g} / \mathrm{m}^{3}$ of "The maintenance and management guideline for air quality within school buildings" recommended by the environmental health law's enforcement regulation under the Ministry of Education and Human Resources Development.

The VOCs and Aldehyde concentration revealed a decreasing trend from the indoor classroom, hallway to outdoor classroom. The overall mean concentration of acetaldehyde was $12.77 \mu \mathrm{g} / \mathrm{m}^{3}$. However, the indoor air-related guidelines for acetaldehyde have not yet been established school in Korea.

Individual VOCs have not yet been designated under the environmental health law of the Ministry of Education and Human Resources Development. Instead, the Ministry of Environment's public facilities guideline was applied. According to these guidelines, 17 to $27 \%$ of tested schools have exceeded given level of benzene, toluene, and xylene; whereas, ethylbenzene and styrene were found to meet the guideline (Table 2).

The indoor and outdoor VOCs concentration in primary schools located in Seoul, Gyeonggi, and Incheon were higher than primary schools in other areas (regional communities, Eup, Myun units). It is likely that the contamination at these schools is the result of industrial activities and high traffic volume. The occurrence and concentrations of VOCs in homes can be affected by outdoor atmospheric conditions, indoor sources, indoor volume, human activities, ventilation rates, and seasonal factors. Furthermore, VOC concentration can vary due to temperature changes, and humidity (Van der wal et al., 1997). A number of investigators have demonstrated that the indoor concentrations of VOC in dwellings are usually higher than outdoor concentration (Jo et al., 2004; Wilson et al., 1993; Sheldon et al., 1991).

This result is possibly due to the influence of other

Table 2. Concentration of target compounds.

\begin{tabular}{|c|c|c|c|c|c|c|c|c|c|}
\hline \multirow[b]{2}{*}{ Compound } & \multicolumn{2}{|c|}{ Classroom $(\mathrm{N}=80)$} & \multicolumn{2}{|c|}{ Outdoor $(\mathrm{N}=80)$} & \multicolumn{2}{|c|}{ Hallway $(\mathrm{N}=80)$} & \multirow{2}{*}{$\begin{array}{l}\mathrm{I} / \mathrm{O} \\
\text { ratio }\end{array}$} & \multirow{2}{*}{$\begin{array}{l}\text { Over value } \\
\text { rate }(\%)\end{array}$} & \multirow{2}{*}{$\begin{array}{c}\text { Reference } \\
\text { value }\end{array}$} \\
\hline & $\begin{array}{c}\text { Mean } \pm \text { S.D } \\
(\text { Min-Max) }\end{array}$ & $\begin{array}{c}\text { Detection } \\
\text { rate }(\%)\end{array}$ & $\begin{array}{c}\text { Mean } \pm \text { S.D } \\
\text { (Min-Max) }\end{array}$ & $\begin{array}{l}\text { Detection } \\
\text { rate }(\%)\end{array}$ & $\begin{array}{l}\text { Mean } \pm \text { S.D } \\
\text { (Min-Max) }\end{array}$ & $\begin{array}{l}\text { Detection } \\
\text { rate }(\%)\end{array}$ & & & \\
\hline Formaldehyde & $\begin{array}{l}22.07 \pm 15.62 \\
(1.12-107.14)\end{array}$ & 100 & $\begin{array}{l}6.12 \pm 7.26 \\
\text { (N.D-49.21) }\end{array}$ & 91 & $\begin{array}{c}10.52 \pm 7.11 \\
\text { (N.D-37.21) }\end{array}$ & 99 & 3.6 & 1.2 & $100^{\mathrm{a}}$ \\
\hline Acetaldehyde & $\begin{array}{l}12.77 \pm 17.61 \\
(1.59-121.06)\end{array}$ & 100 & $\begin{array}{l}6.12 \pm 7.26 \\
\text { (N.D-85.55) }\end{array}$ & 94 & $\begin{array}{l}10.52 \pm 7.11 \\
(1.06-89.39)\end{array}$ & 100 & 1.1 & - & - \\
\hline Benzene & $\begin{array}{c}14.03 \pm 19.38 \\
\text { (N.D-96.64) }\end{array}$ & 96 & $\begin{array}{c}11.59 \pm 15.58 \\
\text { (N.D-68.28) }\end{array}$ & 90 & $\begin{array}{l}12.88 \pm 16.34 \\
\text { (N.D-73.82) }\end{array}$ & 93 & 1.2 & 17 & $30^{\mathrm{b}}$ \\
\hline Toluene & $\begin{array}{l}81.17 \pm 110.61 \\
\text { (N.D-816.47) }\end{array}$ & 97 & $\begin{array}{l}52.99 \pm 66.99 \\
\text { (N.D-261.53) }\end{array}$ & 97 & $\begin{array}{l}74.82 \pm 87.33 \\
\text { (N.D-534.20) }\end{array}$ & 99 & 1.5 & 2.6 & $260^{\mathrm{b}}$ \\
\hline Ethylbenzene & $\begin{array}{l}28.33 \pm 38.51 \\
\text { (N.D-158.58) }\end{array}$ & 95 & $\begin{array}{l}19.27 \pm 30.14 \\
\text { (N.D-116.79) }\end{array}$ & 96 & $\begin{array}{l}24.49 \pm 32.32 \\
\text { (N.D-125.33) }\end{array}$ & 96 & 1.4 & 0.0 & $1000^{\mathrm{b}}$ \\
\hline Xylene & $\begin{array}{l}50.15 \pm 70.74 \\
\text { (N.D-268.74) }\end{array}$ & 87 & $\begin{array}{l}30.94 \pm 50.39 \\
\text { (N.D-191.94) }\end{array}$ & 82 & $\begin{array}{l}46.04 \pm 67.59 \\
\text { (N.D-384.69) }\end{array}$ & 91 & 1.6 & 24 & $100^{\mathrm{b}}$ \\
\hline Styrene & $\begin{array}{r}9.95 \pm 25.14 \\
(\text { N.D-150.58) }\end{array}$ & 64 & $\begin{array}{l}3.86 \pm 9.80 \\
\text { (N.D-61.40) }\end{array}$ & 59 & $\begin{array}{l}6.37 \pm 15.21 \\
(\text { N.D-87.79) }\end{array}$ & 64 & 2.6 & 0 & $260^{\mathrm{b}}$ \\
\hline PM-10 & $\begin{array}{l}88.06 \pm 54.47 \\
(9.70-358.33)\end{array}$ & 100 & $\begin{array}{l}63.91 \pm 41.31 \\
(12.50-179.17)\end{array}$ & 100 & $\begin{array}{l}84.32 \pm 53.66 \\
\text { (N.D-280.15) }\end{array}$ & 98 & 1.4 & 27 & $100^{\mathrm{a}}$ \\
\hline MTBE & $\begin{array}{c}2.18 \pm 5.63 \\
\text { (N.D-32.97) }\end{array}$ & 23 & $\begin{array}{l}3.46 \pm 9.17 \\
\text { (N.D-46.12) }\end{array}$ & 26 & $\begin{array}{r}1.28 \pm 2.90 \\
\text { (N.D-15.33) }\end{array}$ & 25 & 0.6 & - & - \\
\hline
\end{tabular}

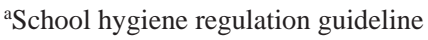

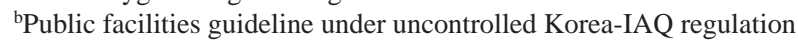


unidentified sources of VOCs from the industrial complex and indoor. The ratio of the indoor (I) to the outdoor (O) air concentration of different compounds, i.e. the $\mathrm{I} / \mathrm{O}$ value, reflects the importance of outdoor versus indoor sources even better than the absolute concentration (Ilgen et al., 2001). This study could be interpreted as an indication of weak indoor sources (I/O values are greater than 1) for this compound (Table 2).

However, the mechanisms by which fine particulate and the other traffic-related pollutants might influence allergen handling by the individual and promote allergic sensitisation and morbidity can be only hypothesised. Diesel particles have been shown to enhance inflammatory reactions and sensitization (Mastrangelo et al., 2003). Like many other industries, the dyeing industry is associated with VOCs emissions from the solvents employed in the dyeing and finishing processes (Wicks Jr. et al., 1994). Of course, other unidentified potential VOC sources could still complicate the distance factor from the nearby roadway and school.

\section{3 Relationship between Indoor Air Quality and Environmental Disease}

In this study, only the survey data obtained from schools were utilized to determine the relationship between the indoor air quality and environmental dis- eases (Tables 3-5). The ISAAC survey results were based on 80 schools since the overall results showed similar patterns (data not shown).

80 primary schools were divided into the upper and lower 40 primary schools with a higher and lower ratio of children who had allergic disease symptoms for the past year. Schools with a higher and lower ratio of students who had symptoms of asthma and atopic dermatitis last year did not have significantly different concentration of aldehydes (formaldehyde and acetaldehyde), VOCs (benzene, toluene, ethylbenzene, xylene), or $\mathrm{PM}_{10}$.

Depending on the measurement site (indoor, hallway, outdoor), the concentration showed expected trend between the upper and lower 40 schools. Good air quality in classrooms and hallways benefits children in their learning ability, helps keep teachers and staff productive, and also is beneficial to their health (Mendell and Heath, 2005; USEPA, 1996). Nevertheless, the variance of each measured concentration was large; thus revealing no statistically significant differences in the concentrations of substances between the upper and lower schools (Table 3). Four volatile organic compounds (BTEX) had higher concentrations in the upper 40 schools. The concentrations of these four materials were also significantly different between

Table 3. Analysis of indoor air value of school with asthma and atopy dermatitis.

(Unit: $\mu \mathrm{g} / \mathrm{m}^{3}$ )

\begin{tabular}{|c|c|c|c|c|c|c|}
\hline & \multicolumn{3}{|c|}{ Asthma } & \multicolumn{3}{|c|}{ Atopy dermatitis } \\
\hline & $\begin{array}{l}\text { Case school } \\
\quad(n=40)\end{array}$ & $\begin{array}{l}\text { Control school } \\
\quad(n=40)\end{array}$ & $\begin{array}{c}\text { p- } \\
\text { value }^{\mathrm{a}}\end{array}$ & $\begin{array}{l}\text { Case school } \\
(n=40)\end{array}$ & $\begin{array}{l}\text { Control school } \\
\quad(n=40)\end{array}$ & $\begin{array}{c}\text { p- } \\
\text { value }^{\mathrm{a}}\end{array}$ \\
\hline Formaldehyde-Classroom & $22.61 \pm 17.9$ & $22.06 \pm 13.4$ & 0.8806 & $24.64 \pm 11.5$ & $19.87 \pm 19.3$ & 0.1968 \\
\hline Formaldehyde-Hallway & $10.34 \pm 6.7$ & $10.99 \pm 7.8$ & 0.6945 & $11.69 \pm 6.5$ & $9.54 \pm 7.8$ & 0.1924 \\
\hline Formaldehyde-Outdoor & $5.63 \pm 4.6$ & $6.92 \pm 9.5$ & 0.4568 & $5.92 \pm 5.3$ & $6.60 \pm 9.2$ & 0.6953 \\
\hline Acetaldehyde-Classroom & $12.20 \pm 20.5$ & $12.49 \pm 17.7$ & 0.9468 & $13.47 \pm 17.8$ & $11.15 \pm 20.5$ & 0.5947 \\
\hline Acetaldehyde-Hallway & $8.27 \pm 11.7$ & $12.59 \pm 20.2$ & 0.2551 & $11.28 \pm 16.5$ & $9.42 \pm 16.5$ & 0.6192 \\
\hline Acetaldehyde-Outdoor & $10.42 \pm 18.9$ & $12.95 \pm 21.6$ & 0.5838 & $9.55 \pm 16.6$ & $13.86 \pm 23.4$ & 0.3537 \\
\hline Benzene-Classroom & $11.84 \pm 16.7$ & $16.22 \pm 21.8$ & 0.3276 & $12.20 \pm 18.0$ & $15.76 \pm 20.6$ & 0.4278 \\
\hline Benzene-Hallway & $11.57 \pm 15.5$ & $14.12 \pm 17.2$ & 0.4998 & $11.88 \pm 15.9$ & $13.88 \pm 16.8$ & 0.5956 \\
\hline Benzene-Outdoor & $13.76 \pm 19.0$ & $9.37 \pm 10.8$ & 0.2293 & $11.58 \pm 16.4$ & $11.61 \pm 14.9$ & 0.9926 \\
\hline Toluene-Classroom & $92.05 \pm 140.9$ & $70.29 \pm 68.4$ & 0.3960 & $72.61 \pm 69.0$ & $89.30 \pm 139.6$ & 0.5083 \\
\hline Toluene-Hallway & $82.42 \pm 103.1$ & $67.60 \pm 69.8$ & 0.4681 & $64.73 \pm 68.1$ & $84.90 \pm 103.1$ & 0.3179 \\
\hline Toluene-Outdoor & $62.82 \pm 76.3$ & $42.89 \pm 55.1$ & 0.2061 & $48.93 \pm 68.8$ & $57.16 \pm 65.7$ & 0.6033 \\
\hline Ethylbenzene-Classroom & $26.33 \pm 36.2$ & $30.33 \pm 41.0$ & 0.6534 & $28.87 \pm 40.4$ & $27.81 \pm 37.2$ & 0.9052 \\
\hline Ethylbenzene-Hallway & $23.32 \pm 28.7$ & $25.59 \pm 35.7$ & 0.7611 & $22.92 \pm 30.2$ & $26.05 \pm 34.6$ & 0.6757 \\
\hline Ethylbenzene-Outdoor & $23.57 \pm 34.3$ & $14.85 \pm 24.9$ & 0.2191 & $19.02 \pm 32.7$ & $19.53 \pm 27.7$ & 0.9440 \\
\hline Xylene-Classroom & $41.09 \pm 55.9$ & $59.44 \pm 83.0$ & 0.2546 & $45.35 \pm 66.2$ & $55.07 \pm 75.7$ & 0.5449 \\
\hline Xylene-Hallway & $44.57 \pm 70.3$ & $47.54 \pm 65.6$ & 0.8463 & $37.64 \pm 48.8$ & $54.65 \pm 82.4$ & 0.2701 \\
\hline Xylene-Outdoor & $36.56 \pm 55.4$ & $25.18 \pm 44.6$ & 0.3188 & $30.06 \pm 51.8$ & $31.84 \pm 49.5$ & 0.8764 \\
\hline $\mathrm{PM}_{10}$-Classroom & $89.32 \pm 39.9$ & $87.37 \pm 68.3$ & 0.8790 & $94.46 \pm 58.5$ & $81.95 \pm 51.6$ & 0.3207 \\
\hline $\mathrm{PM}_{10}$-Hallway & $92.14 \pm 59.2$ & $75.20 \pm 45.9$ & 0.2605 & $90.78 \pm 47.9$ & $74.79 \pm 61.2$ & 0.2964 \\
\hline $\mathrm{PM}_{10}$-Outdoor & $68.91 \pm 42.2$ & $58.07 \pm 40.3$ & 0.3508 & $71.81 \pm 45.2$ & $52.24 \pm 32.3$ & 0.0941 \\
\hline
\end{tabular}


Table 4. Analysis of indoor air value of school with allergic rhinitis and conjunctivitis.

(Unit: $\mu \mathrm{g} / \mathrm{m}^{3}$ )

\begin{tabular}{|c|c|c|c|c|c|c|}
\hline & \multicolumn{3}{|c|}{ Allergic rhinitis } & \multicolumn{3}{|c|}{ Allergic conjunctivitis } \\
\hline & $\begin{array}{l}\text { Case school } \\
\quad(n=40)\end{array}$ & $\begin{array}{l}\text { Control school } \\
\quad(\mathrm{n}=40)\end{array}$ & $\begin{array}{c}\text { p- } \\
\text { value }^{\mathrm{a}}\end{array}$ & $\begin{array}{l}\text { Case school } \\
\quad(n=40)\end{array}$ & $\begin{array}{l}\text { Control school } \\
\quad(\mathrm{n}=40)\end{array}$ & $\begin{array}{c}\text { p- } \\
\text { value }^{\mathrm{a}}\end{array}$ \\
\hline Formaldehyde-Classroom & $23.87 \pm 18.4$ & $20.86 \pm 12.8$ & 0.4105 & $25.74 \pm 18.2$ & $18.67 \pm 11.9$ & 0.0460 \\
\hline Formaldehyde-Hallway & $11.70 \pm 6.0$ & $9.63 \pm 8.1$ & 0.2083 & $10.97 \pm 5.2$ & $10.32 \pm 8.9$ & 0.7000 \\
\hline Formaldehyde-Outdoor & $5.91 \pm 4.4$ & $6.58 \pm 9.5$ & 0.6948 & $7.22 \pm 8.5$ & $5.19 \pm 5.9$ & 0.2250 \\
\hline Acetaldehyde-Classroom & $13.96 \pm 21.8$ & $10.72 \pm 16.0$ & 0.4559 & $15.61 \pm 24.8$ & $8.90 \pm 9.2$ & 0.1167 \\
\hline Acetaldehyde-Hallway & $11.42 \pm 17.5$ & $9.33 \pm 15.4$ & 0.5772 & $11.83 \pm 17.1$ & $8.84 \pm 15.8$ & 0.4259 \\
\hline Acetaldehyde-Outdoor & $12.43 \pm 19.9$ & $10.87 \pm 20.7$ & 0.7352 & $11.59 \pm 19.8$ & $11.72 \pm 20.8$ & 0.9778 \\
\hline Benzene-Classroom & $20.01 \pm 23.3$ & $8.35 \pm 12.5$ & 0.0094 & $16.79 \pm 22.1$ & $11.41 \pm 16.2$ & 0.2289 \\
\hline Benzene-Hallway & $18.81 \pm 19.1$ & $6.63 \pm 9.6$ & 0.0008 & $13.71 \pm 17.6$ & $12.00 \pm 15.1$ & 0.6517 \\
\hline Benzene-Outdoor & $15.00 \pm 16.9$ & $8.45 \pm 13.7$ & 0.0724 & $13.08 \pm 16.7$ & $10.07 \pm 14.4$ & 0.4135 \\
\hline Toluene-Classroom & $111.00 \pm 139.5$ & $52.88 \pm 63.2$ & 0.0247 & $78.57 \pm 69.3$ & $83.65 \pm 139.9$ & 0.8405 \\
\hline Toluene-Hallway & $104.09 \pm 96.7$ & $43.97 \pm 64.1$ & 0.0021 & $67.61 \pm 61.7$ & $82.41 \pm 108.4$ & 0.4706 \\
\hline Toluene-Outdoor & $67.55 \pm 74.6$ & $39.58 \pm 56.8$ & 0.0744 & $52.58 \pm 64.8$ & $53.41 \pm 70.1$ & 0.9584 \\
\hline Ethylbenzene-Classroom & $40.33 \pm 42.3$ & $16.94 \pm 30.9$ & 0.0073 & $35.92 \pm 43.5$ & $21.12 \pm 31.9$ & 0.0941 \\
\hline Ethylbenzene-Hallway & $36.05 \pm 35.7$ & $12.29 \pm 23.1$ & 0.0009 & $27.93 \pm 35.3$ & $20.86 \pm 28.9$ & 0.3434 \\
\hline Ethylbenzene-Outdoor & $26.89 \pm 32.8$ & $12.25 \pm 25.9$ & 0.0371 & $21.62 \pm 31.3$ & $16.86 \pm 29.2$ & 0.5034 \\
\hline Xylene-Classroom & $63.47 \pm 70.9$ & $36.49 \pm 68.8$ & 0.0902 & $59.49 \pm 78.5$ & $40.57 \pm 61.3$ & 0.2371 \\
\hline Xylene-Hallway & $68.36 \pm 76.5$ & $23.14 \pm 47.9$ & 0.0024 & $48.05 \pm 57.5$ & $43.97 \pm 77.3$ & 0.7901 \\
\hline Xylene-Outdoor & $41.76 \pm 54.7$ & $19.84 \pm 43.5$ & 0.0526 & $34.91 \pm 52.9$ & $26.86 \pm 48.0$ & 0.4813 \\
\hline $\mathrm{PM}_{10}$-Classroom & $98.56 \pm 70.6$ & $77.64 \pm 29.2$ & 0.0906 & $103.63 \pm 70.5$ & $72.29 \pm 24.2$ & 0.0108 \\
\hline $\mathrm{PM}_{10}$-Hallway & $95.27 \pm 49.3$ & $68.16 \pm 56.3$ & 0.0735 & $102.39 \pm 55.9$ & $59.69 \pm 39.7$ & 0.0036 \\
\hline $\mathrm{PM}_{10}$-Outdoor & $74.37 \pm 46.9$ & $48.46 \pm 24.9$ & 0.0130 & $76.31 \pm 48.3$ & $46.99 \pm 20.1$ & 0.0047 \\
\hline
\end{tabular}

${ }^{\mathrm{a}} \mathrm{T}$-test

classrooms and hallways $(\mathrm{p}<0.05)$. In other words, schools where many students had allergic rhinitis symptoms in the prior year had higher concentrations of VOCs compared to schools where fewer students had allergic rhinitis symptoms. Concentration of particulate matter was higher in schools where majority of students showed allergic symptoms. However, significant differences were only found in the indoor and outdoor environment (Table 4).

Indoor formaldehyde and $\mathrm{PM}_{10}$ levels in classroom were significantly $(\mathrm{p}<0.05)$ higher in schools with high proportion of allergic conjunctivitis compared to schools with lower proportion. The concentrations of other substances were also higher at schools where many children had allergic conjunctivitis symptoms, but this difference was not significant. Only $\mathrm{PM}_{10}$ measured outdoors was significantly $(\mathrm{p}<0.05)$ different between the upper and the lower $25 \%$ schools (20 schools). The concentration of contaminant in the air for the upper $25 \%$ of schools was higher than the concentration at the lower $25 \%$ of schools (Table 5).

Previous studies have shown that air pollutants related to vehicles can stimulate allergies (D'Amato et al., 2000; Takenaka et al., 1995). In vitro experiment of Gilmour (Gilmour, 1995) also demonstrated that diesel combustion substances could increase immunoglobulin $\mathrm{E}(\mathrm{IgE})$ levels. These associations reflect the shared inflammation process underlying allergic rhinitis and asthma, and potentially explain the frequent co-existence of these disorders. These findings may be the result of an unhealthy environment at public schools, as well as different socio-economic status leading to diverse behavioural and environmental factors (ISAAC Steering Committee, 1998; Venn et al., 1998).

It is worth noting that these allergic diseases can result not only from exposure to indoor/outdoor harmful chemical substances, but also from exposure to micro-organisms, house dust mites, and fungi (Nafstad et al., 2005; De Marco et al., 2004). In addition, lifestyle factors such as dietary habits, breast feeding, atopy heritability, month of birth, parent smoking, and sex have all been reported to play an important role in causing allergic sensitivity (Ford, 2005; Monteil et al., 2004; Sly, 1999; Tariq et al., 1998). Because this study analyzed cross-sectional data, it cannot be determined whether the onset of allergy disease followed the onset of indoor air quality. Prospective data are needed to determine if such an association is causal and if there is a time frame during which a child is at increased risk for the development of allergic disease. 
Table 5. Analysis of indoor air value of school with asthma and atopy dermatitis.

(Unit: $\mu \mathrm{g} / \mathrm{m}^{3}$ )

\begin{tabular}{|c|c|c|c|c|c|c|}
\hline & \multicolumn{3}{|c|}{ Asthma } & \multicolumn{3}{|c|}{ Atopy dermatitis } \\
\hline & $\begin{array}{l}\text { Case school } \\
\quad(\mathrm{n}=20)^{\mathrm{a}}\end{array}$ & $\begin{array}{l}\text { Control school } \\
\qquad(\mathrm{n}=20)^{\mathrm{b}}\end{array}$ & $\begin{array}{c}\text { p- } \\
\text { value }^{c}\end{array}$ & $\begin{array}{l}\text { Case school } \\
\quad(\mathrm{n}=20)^{\mathrm{a}}\end{array}$ & $\begin{array}{l}\text { Control school } \\
(n=20)^{b}\end{array}$ & $\begin{array}{c}\text { p- } \\
\text { value }^{c}\end{array}$ \\
\hline Formaldehyde-Classroom & $27.73 \pm 22.5$ & $17.32 \pm 9.8$ & 0.0705 & $24.77 \pm 11.6$ & $19.09 \pm 13.8$ & 0.1764 \\
\hline Formaldehyde-Hallway & $12.08 \pm 7.3$ & $10.95 \pm 8.2$ & 0.6499 & $10.96 \pm 4.9$ & $9.77 \pm 9.0$ & 0.6201 \\
\hline Formaldehyde-Outdoor & $6.41 \pm 5.6$ & $6.90 \pm 11.7$ & 0.8708 & $5.56 \pm 3.4$ & $6.67 \pm 7.5$ & 0.5680 \\
\hline Acetaldehyde-Classroom & $16.88 \pm 28.3$ & $7.81 \pm 3.8$ & 0.1712 & $9.63 \pm 3.6$ & $9.68 \pm 13.1$ & 0.9875 \\
\hline Acetaldehyde-Hallway & $11.17 \pm 15.9$ & $6.48 \pm 4.1$ & 0.2152 & $8.30 \pm 10.7$ & $5.27 \pm 3.7$ & 0.2452 \\
\hline Acetaldehyde-Outdoor & $11.16 \pm 20.7$ & $7.74 \pm 14.0$ & 0.5521 & $7.98 \pm 13.3$ & $10.59 \pm 20.3$ & 0.6388 \\
\hline Benzene-Classroom & $12.98 \pm 19.3$ & $11.72 \pm 12.9$ & 0.8165 & $12.62 \pm 19.2$ & $9.05 \pm 11.4$ & 0.4914 \\
\hline Benzene-Hallway & $12.87 \pm 16.6$ & $10.73 \pm 13.4$ & 0.6635 & $10.80 \pm 15.5$ & $9.43 \pm 10.9$ & 0.7590 \\
\hline Benzene-Outdoor & $12.52 \pm 16.5$ & $9.74 \pm 11.3$ & 0.5558 & $9.88 \pm 14.0$ & $7.19 \pm 7.5$ & 0.4822 \\
\hline Toluene-Classroom & $69.71 \pm 75.2$ & $70.03 \pm 67.5$ & 0.9892 & $77.75 \pm 66.0$ & $101.85 \pm 183.0$ & 0.5946 \\
\hline Toluene-Hallway & $63.04 \pm 59.8$ & $62.77 \pm 62.6$ & 0.9889 & $58.88 \pm 59.5$ & $94.17 \pm 133.0$ & 0.3126 \\
\hline Toluene-Outdoor & $61.22 \pm 79.5$ & $52.35 \pm 58.5$ & 0.7026 & $54.93 \pm 70.6$ & $48.22 \pm 62.5$ & 0.7683 \\
\hline Ethylbenzene-Classroom & $29.16 \pm 42.5$ & $26.54 \pm 36.0$ & 0.8404 & $34.72 \pm 42.9$ & $13.38 \pm 21.6$ & 0.0636 \\
\hline Ethylbenzene-Hallway & $21.89 \pm 24.6$ & $20.22 \pm 33.3$ & 0.8616 & $20.58 \pm 23.9$ & $17.93 \pm 26.8$ & 0.7518 \\
\hline Ethylbenzene-Outdoor & $21.53 \pm 30.3$ & $15.34 \pm 24.2$ & 0.4987 & $16.74 \pm 27.2$ & $11.99 \pm 22.2$ & 0.5756 \\
\hline Xylene-Classroom & $42.21 \pm 59.4$ & $63.69 \pm 92.3$ & 0.3905 & $53.46 \pm 68.5$ & $26.12 \pm 42.1$ & 0.1407 \\
\hline Xylene-Hallway & $39.96 \pm 44.0$ & $40.47 \pm 69.4$ & 0.9779 & $34.91 \pm 39.8$ & $42.57 \pm 91.2$ & 0.7391 \\
\hline Xylene-Outdoor & $34.09 \pm 48.6$ & $26.50 \pm 46.2$ & 0.6202 & $26.36 \pm 42.0$ & $18.93 \pm 39.0$ & 0.5709 \\
\hline $\mathrm{PM}_{10}$-Classroom & $89.39 \pm 45.9$ & $83.62 \pm 65.5$ & 0.7536 & $98.08 \pm 45.0$ & $73.35 \pm 29.5$ & 0.0556 \\
\hline $\mathrm{PM}_{10}$-Hallway & $82.57 \pm 44.3$ & $56.51 \pm 21.8$ & 0.0577 & $96.41 \pm 49.4$ & $71.34 \pm 47.3$ & 0.2237 \\
\hline $\mathrm{PM}_{10}$-Outdoor & $66.75 \pm 37.4$ & $42.64 \pm 18.5$ & 0.0391 & $85.66 \pm 50.8$ & $41.43 \pm 19.6$ & 0.0043 \\
\hline
\end{tabular}

${ }^{\mathrm{a}}$ Level highest $25 \%$, ${ }^{\mathrm{b}}$ Level lowest $25 \%,{ }^{\mathrm{c}} \mathrm{T}$-test

Causal associations cannot be assumed in this crosssectional analysis. A further prospective investigation of the effect of social-economic status among children would be needed. In addition, numerous hypotheses relating to family size, early childhood infections and hygiene, allergen exposure, diet and obesity, and pollution may be involved (Hong et al., 2004).

The concentration results of tested substances used at the current study are the measurement data which did not reflect the various properties of school space. There are limitations of result interpretation. But in the selection of subjected schools, the schools where can represent the characteristics of the selected regions were selected, and made efforts to complement the representativeness by measuring the indoor, hallway and outdoor sites of 80 schools.

\section{CONCLUSIONS}

The International Study of Asthma and Allergies in Childhood (ISAAC) was previously developed to provide an acceptable method of measuring the prevalence of asthma and other atopic diseases in children (Weiland et al., 2004). In this study, prevalence of asthma was found to be $8.0 \%(40,522$ tested). The prevalence of asthma increased from $2.7 \%$ in 1995 to $5.3 \%$ in 2000 . The awareness of asthma by doctors and/or patients and their parents may have increased over this period of time, explaining, at least in part, the increased prevalence of asthma in South Korea (Hong et al., 2004).

This study revealed a trend for higher contaminating substance concentrations at schools with higher number of children with allergic symptoms ("upper schools") compared to schools with fewer reported allergic symptoms ("lower schools"). The study showed no statistically significant differences between the upper and lower school for many of the contaminants due to the large variance. The case was different for most VOCs, in which significantly higher concentrations $(\mathrm{p}<0.05)$ have been found in the upper 40 schools compared to the lower 40 schools. Similarly, schools with many students with allergic rhinitis and conjunctivitis showed high concentrations of $\mathrm{PM}_{10}$. It is predicted that use of first round of measurement data and inconsideration of characteristic features of each school space may have limited the interpretation of the results. However, an effort was made to select schools that are representative of particular regions. Samples were 
also taken from three different areas (indoors, hallways, and outdoors) of schools. whereas cross-sectional allergical studies did not find a relationship between indoor air test performance and duration of disease prevalence.

In addition, long-term follow-up studies should be carried out to investigate related factors even after controlling for potential confounding factors such as age, sex, birth weight, breastfeeding, parental asthma, passive exposure to smoke, socioeconomic status of environmental diseases in school-aged children.

\section{ACKNOWLEDGEMENTS}

This study has been supported (2007) by the Ministry of Education, Science and Technology. We thank the parents and children who have willingly participated in this study.

\section{REFERENCES}

Asher, M.I., Keil, U., Anderson, H.R., Beasley, R., Crane, J., Martinez, F. (1995) International study of asthma and allergies in childhood (ISAAC): rationale and methods. European Respiratory Journal 8, 483-491.

Braniš, M., Rezácová, P., Domasová, M. (2005) The effect of outdoor air and indoor human activity on mass concentrations of $\mathrm{PM}_{10}, \mathrm{PM}_{2.5}$, and $\mathrm{PM}_{1}$ in a classroom. Environmental Research 99, 143-149.

Breysse, P.N., Buckley, T.J., Williams, D., Beck, C.M., Jo, S.J., Merriman, B., Kanchanaraksa, S., Swartz, L.J., Callaha, K.A., Butz, A.M., Rand, C.S., Diette, G.B., Krishnan, J.A., Moseley, A.M., Curtin-Brosnan, J., Durkin, N.B., Eggleston, P.A. (2005) Indoor exposures to air pollutants and allergens in homes of asthmatic children in inner-city Baltimore. Environmental Research 98, 167-176.

Brunekreef, B., Janssen, N.A., de Hartog, J. (1997) Air pollution from truck traffic and lung function in children living near motorways. Epidemiology 8, 298-303.

D’Amato, G., Liccardi, G., D’Amato, M. (2000) Environmental risk factors (outdoor air pollution and climatic changes) and increased trend of respiratory allergy. Journal of Investigational Allergology and Clinical Immunology 10, 123-128.

Delfino, R.J. (2002) Epidemiologic evidence for asthma and exposure to air toxics: linkages between occupational, indoor, and community air pollution research. Environmental Health Perspective 110(Suppl 4), 573-589.

De Marco, R., Pattaro, C., Locatelli, F., Svanes, C. (2004) The ECRHS Study Group. Influence of early life exposures on incidence and remission of asthma throughout life. Journal of Investigational Allergology and Clinical Immunology 113, 845-852.

Downs, S.H., Marks, G.B., Sporik, R., Belosouva, E.G.,
Car, N.G., Peat, J.K. (2001) Continued increase in the prevalence of asthma and atopy. Archives of Disease in Childhood 84, 20-23.

Ford, E.S. (2005) The epidemiology of obesity and asthma. Journal of Investigational Allergology and Clinical Immunology 115, 897-909.

Gilmour, M.I. (1995) Interaction of air pollutants and pulmonary allergic responses in experimental animals. Toxicology 105, 335-342.

Hirsch, T., Weiland, S.K., von Mutius, E., Safeca, A.F., Grafe, H., Csaplovics, E., Duhme, H., Keil, U., Leupold, W. (1999) Inner city air pollution and respiratory health and atopy in children. European Respiratory Journal 14, 669-677.

Hong, S.J., Lee, M.S., Sohn, M.H., Shim, J.Y., Han, Y.S., Park, K.S., Ahn, Y.M., Son, B.K., Lee, H.B. (2004) Korean ISAAC Study Group. Self-reported prevalence and risk factors of asthma among Korean adolescents: 5-year follow-up study, 1995-2000. Clinical Experimental Allergy 34(10), 1556-1562.

Ho, W.C., Hartley, W.R., Myers, L., Lin, M.H., Lin, Y.S., Lien, C.H., Lin, R.S. (2007) Air pollution, weather, and associated risk factors related to asthma prevalence and attack rate. Environmental Research 104, 402409.

Ilgen, E., Karfich, N., Levsen, K., Angerer, J., Schneider, P., Heinrich, J., Wichmann, H., Dunemann, L., Begerow, J. (2001) Aromatic hydrocarbons in the atmospheric environment: part I. Indoor versus outdoor sources, the influence of traffic. Atmospheric Environment 35, 1235-1252.

Jo, W.K., Lee, J.W., Shin, D.C. (2004) Exposure to volatile organic compounds in residences adjacent to dyeing industrial complex. International Archives of Occupational and Environmental Health 77, 113-120.

Knox, R.B., Suphioglu, C., Taylor, P., Desai, R., Watson, H.C., Peng, J.L., Bursill, L.A. (1997) Major grass pollen allergen Lol $\mathrm{p} 1$ binds to diesel exhaust particles: implications for asthma and air pollution. Clinical and Experimental Allergy 27, 246-51.

Lee, S.I., Shin, M.H., Lee, H.B., Lee, J.S., Son, B.K., Koh, Y.Y., Kim, K.E., Ahn, Y.O. (2001) Prevalences of symptoms of asthma and other allergic diseases in Korean children: a nationwide questionnaire survey. Journal of Korean Medical Science 16, 155-164.

The Ministry of Health and Welfare (MOHW) (2005) Korea National Health and Nutrition Examination Survey.

Mastrangelo, G., Clonfero, E., Pavznerllo, S., Fedeli, U., Fadda, E., Turato, A., Piccinni, S., Montagnani, R., Marcer, G. (2003) Exposure to diesel exhaust enhances total $\mathrm{IgE}$ in non-atopic dockers. International Archives of Occupational and Environmental Health 76, 63-68.

Mendell, M.J., Heath, G.A. (2005) Do indoor pollutants and thermal conditions in schools influence student perfor-mance? A critical review of the literature. Indoor Air 15, 27-52.

Monteil, M.A., Joseph, G., Chang, C., Kit, G., Wheeler, 
R.M., Antoine. (2004) Smoking at home is strongly associated with symptoms of asthma and rhinitis in children of primary school age in Trinidad and Tobago. Revista Panamericana De Salud Publica 16, 193-198.

Nafstad, P., Brunekreef, B., Skrondal, A., Nystad, W. (2005) Early respiratory infections, asthma, and allergy: 10-year follow-up of the Oslo Birth Cohort. Pediatrics $116,255-262$.

Nicolai, T., Carr, D., Weiland, S.K., Duhme, H., von Ehrenstein, O., Wagner, C., von Mutius, E. (2003) Urban traffic and pollutant exposure related to respiratory outcomes and atopy in a large sample of children. European Respiratory Journal 21, 956-963.

Nielsen, G.D., Hansen, J.S., Lund, R.M., Bergqvist, M., Larsen, S.T., Clausen, S.K., Thygesen, P., Poulsen, O.M. (2002) IgE-mediated asthma and rhinitis I: a role of allergen exposure? Pharmacology Toxicology 90, 231-242.

Nielsen, G.D., Olsen, O., Larsen, S.T., Løvik, M., Poulsen, L.K., Glue, C., Brandorff, N.P., Nielsen, P.J. (2005) IgE-mediated sensitisation, rhinitis and asthma from occupational exposures. Smoking as a model for airborne adjuvants? Toxicology 216, 87-105.

Oh, J.W., Kim, G.E., Pyun, B.Y., Lee, H.L., Choung, J.T., Hong, S.J., Park, K.S., Lee, S.Y., Song, S.W., Kim, C.H., Ahn, K.W., Nam, S.Y., Shon, M.H., Kim, W.K., Lee, M.H., Kwon, B.C., Chio, S.Y., Lee, H.B., Lee, S.I., Lee, J.S. (2003) Nationwide study for epidemiological change of atopic dermatitis in school aged children between 1995 and 2000 and kindergarten aged children in 2003 in Korea. Pediatric Allergy and Respiratory Disease 13, 227-237.

Pandya, R.J., Solomon, G., Kinner, A., Balmes, J.R. (2002) Diesel exhaust and asthma: hypotheses and molecular mechanisms of action. Environmental Health Perspective 110(Suppl. 1), 103-112.

Popp, W., Zwick, H., Steyner, K., Rauscher, H., Wanke, T. (1989) Sensitization to aeroallergens depends on envionmental factors. Allergy 44, 572-575.

Sheldon, L.S., Clayton, A., Jones, B., Keever, J., Perritt, R., Smith, D., Whitaker, D., Whitmore, R. (1991) Indoor pollutant concentrations and exposures. Final Report, California Air Resources Board, Sacramento.

Silvers, A., Florence, B.T., Rourke, D.L., Lorimor, R.J. (1994) How children spend their time-a sample survey for use in exposure and risk assessment. Risk Analysis 14(6), 931-944.

Siskos, P.A., Bouba, K.E., Stroubou, A.P. (2001) Determination of selected pollutants and measurement of physical parameters for the evaluation of indoor air quality in school buildings in Athens, Greece. Indoor and Built Environment 10(3-4), 185-192.

Sly, R.M. (1999) Changing prevalence of allergic rhinitis and asthma. Annals of Allergy Asthma and Immunology 82, 233-248.

Strachen, D., Gerritsen, J. (1996) Long-term outcome of early childhood wheezing population data. European Respiratory Journal 21, 42-47.
Takenaka, H., Zhang, K., Diaz-Sanchez, D. 1995. Enhanced human IgE production results from exposure to the aromatic hydrocarbons from diesel exhaust: direct effects on B-cell IgE production. Journal of Investigational Allergology and Clinical Immunology 95(1 Part 1), 103-115.

Tariq, S.M., Matthews, S.M., Hakim, E.A., Stevens, M., Arshad, S.H., Hide, D.W. (1998) The prevalence of and risk factors for atopy in early childhood: a whole population birth cohort study. Journal of Allergy Clinical Immunology 101, 587-593.

The International Study of Asthma and Allergies in Childhood (ISAAC) Steering Committee (1998) Worldwide variations in the prevalence of asthma symptoms: the International Study of Asthma and Allergies in Childhood (ISAAC). European Respiratory Journal 12, 315335.

USEPA (1996) Indoor Air Quality in Schools, Washington, DC. Unites States Environmental Protection Agency, Washington.

Van der wal, J.F., Hoogeveen, A.W., Wouda, P. (1997) The influence of temperature on the emission of volatile organic compounds from PVC flooring, carpet, and paint. Indoor Air 7, 215-221.

Venn, A., Lewis, S., Cooper, M., Hill, J., Britton, J. (1998) Questionnaire study of effect of sex and age on the prevalence of wheeze and asthma in adolescence. British Medical Journal 316, 1945-1946.

Weiland, S.K., Bjorksten, B., Brunecreef, B., Cookson, W.O.C., Von Mutius, E., Strachan, D.P. (2004) The international study of asthma and allergies in childhood phase II study group. Phase II of the International Study of Asthma and Allergies in Childhood (ISAAC II): rationale and methods. European Respiratory Journal 24, 406-412.

Weiland, S.K., Mundt, K.A., Ruckmann, A., Keil, U. (1994) Self-reported wheezing and allergic rhinitis in children and traffic density on street of residence. Annals Epidemiology 4, 243-247.

Wicks, Jr., Zeno, W., Jones, F.N., Pappas, S.P. (1994) Organic coatings: Science and Technology, Vol. 1. Wiley, Inc., New York, pp. 229-266.

Wilson, A.L., Colome, S.D., Tian, Y. (1993) California Residential Indoor Air Quality Study. Vol. 1: Methodology and Descriptive Statistics. Integrated Environmental Services, Irvine, CA.

Wyler, C., Braun-Fahrlander, C., Kunzli, N. (2000) Exposure to motor vehicle traffic and allergic sensitization. The Swiss Study on Air Pollution and Lung Diseases in Adults (SAPALDIA) team. Epidemiology 11(4), 450-456.

Yeon, N.S., Sun, Y.H., Kyung, K.W. (2005) Prevalence of allergic disease in kindergarten age children in Korea. Pediatric Allergy and Respiratory Disease 15, 439445.

(Received 8 May 2010, accepted 14 October 2010) 